\title{
An inexpensive system for measuring the processing of relational information by romantic partners
}

\author{
ARMAND DE CLERCQ and ANN BUYSSE \\ University of Ghent, Ghent, Belgium
}

\begin{abstract}
The present paper describes the computer aspects of a reaction time experiment with couples. The hardware consists of two computers connected through a local area network. Issues that deal with the first PC include timing routines, screen control, mice data decoding, and synchronization of computer software-written in Assembler for a PC without hard disk-with information presented in a videotape. The second computer was used for data processing that was written in VBA. Although the system was created for a dedicated purpose, it is easily applicable to other environments.
\end{abstract}

The present paper discusses the computer aspects of a reaction time (RT) experiment with couples. The major research question addressed in the experiment was how information about one's marital relationship is perceived, interpreted, stored, and recalled. The experiment, including theoretical underpinnings, methodological details, and results, is described in Buysse, De Clercq, Roeyers, and Verhofstadt (1999). In short, heterosexual couples in a large Belgian city were asked to voluntarily participate in an extended couple investigation. Fifty-six couples were invited to the laboratory and exposed to videotape information that represented their marital relationships. They were simultaneously asked to complete a choice reaction time (CRT) task in a dual-task paradigm. The present article discusses the computer aspects of the design, implementation, and coordination of this dual-task experiment for couples, especially those associated with the CRT task.

In the CRT task, both spouses had to respond to $150 \mathrm{X}$ or $\mathrm{O}$ stimuli. A warning stimulus (**), $50 \mathrm{msec}$ in duration, that appeared in the center of the screen signaled the beginning of each trial. This warning stimulus signaled the subjects to press the center home button of the threebutton Logitech mouse with the index finger of the dominant hand. The subjects were further instructed to keep their finger on the home button until the target stimulus was presented and then to press the left button with the finger if the target stimulus was an $\mathrm{X}$ and the right button if the target stimulus was an O. Congruent with Stelmack, Houlihan, and McGarry-Roberts (1993), the target stimulus was presented in the center of the screen for a duration of $1,600 \mathrm{msec}$ at random intervals of $640-2,640 \mathrm{msec}$ from the onset of the warning stimulus. The duration of each trial, from the onset of the target stimulus to the onset

Correspondence concerning this article should be addressed to A. De Clercq, Department of Applied Mathematics and Computer Science, University of Ghent, Krijgslaan 281 S9, B9000 Ghent, Belgium (email: armand.declercq@rug.ac.be). of the warning stimulus of the next trial, varied randomly between 3 and $5 \mathrm{sec}$. The two target stimuli were presented 75 times each in a random order. Two dependent measures were computed from this CRT task. Following Jensen and Munro (1979), RT was defined as the time from the onset of the target stimulus to the release of the home button. Movement time (MT) was defined as the time from the release of the home button to the onset of the left or the right buttonpress. A practice period of 10 trials ensured that the subjects understood the procedures before performing the tasks. The experimenter, but not the couple, was able to follow the performances of both partners on a computer screen. In case the experimenter judged that the subjects did not completely understand the task, a third period of 10 trials was given.

\section{EXPERIMENTAL CONCEPT}

The present dual-task experiment required that audiovisual material from a videotape be presented simultaneously with the CRT task stimuli. Because of this, we could not use standard software and/or equipment in which a computer is used to display stimuli, without support for the simultaneous projection of a video in full screen. In addition to the lack of available software, most Pentium PCs are not able to handle full-screen video, especially not in combination with millisecond accuracy. We therefore decided to use a video recorder connected to a projector to display the videotape and a small 486 PC connected to a second projector to display the CRT task stimuli. Both projectors were focused on the same large viewing screen ( $4 \times 4 \mathrm{~m}$, located $6 \mathrm{~m}$ in front of the table at which the couple was seated). The male and female partners of a couple were situated next to one another, approximately 2 in. apart, in order to ensure that both partners received all the visual and auditory information from the same perspective.

We predicted that the time necessary to react to the CRT task stimuli would systematically vary with the spe- 
cific information presented in the videotape. It was therefore necessary to synchronize the video with the computer that generated the CRT task stimuli. We solved this problem by putting a short beep $(750 \mathrm{~Hz})$ at the beginning of the videotape. This signal was then connected with one of the input lines of the parallel port of the PC.

Two serial ports of the PC were further used for the connection of two Logitech mice (one for each partner). It was therefore necessary to place the computer next to the partners, which made it impossible for the experimenter to control the experiment with this computer. For this reason, and because programming the $\mathrm{PC}$ hardware for handling timing, fast stimulus projection, and direct mouse data is most easily done in a DOS program, we decided to use two PCs. The first PC is a 486 PC, which we will further refer to as the SlavePC. It only runs an MSDOS program that controls the stimulus display, the recording of mouse presses with millisecond accuracy, and the synchronization of the stimuli display with the start of the video. The second PC-called the MasterPC-is connected to the SlavePC through a local area network.

The MasterPC runs Windows 95 or Windows NT. All the software for controlling the experiment and data processing can be done with the MasterPC, and all state-ofthe-art programming tools can be used. An additional advantage is that the MasterPC can be located out of the sight of the couple. A simple protocol that makes use of dummy files as the locking semaphore is used for synchronizing both PCs (see below). After each session, a brief description is displayed on the MasterPC concerning the percentage of correct mouse clicks and the various incorrect responses of the subjects (mouse buttons clicked too early, wrong response, etc.). As such, it was possible to check whether both spouses completely understood the task and used the mouse in the intended way. Because the subjects themselves were not able to watch the results on the MasterPC screen, they could not be influenced by these results or compare their own results with those of their partner.

The idea of using a small dedicated computer that is connected with a larger computer for the purpose of data gathering is not new. Walter and Palya (1984), for example, described a similar system as long ago as 1984 and elaborated on it in their later publications (Palya \& Walter, 1993). Most systems described in the literature use a special card placed inside the PC or are connected with the PC via the parallel or serial communication port (Palya, Walter, \& Chu, 1995). Although this method is suitable, we used an old 486 PC with an ethernet card but without a hard disk. The old 386 or 486 PCs have little market value but were just what was needed in the present experiment as a cheap replacement for a dedicated controller.

\section{REQUIRED HARDWARE AND COMMERCIAL SOFTWARE}

Besides two computer/video projectors, the required hardware consists of a 486 PC without a hard disk, a PC
( 486 or better) in standard configuration for running Windows 95 , two Logitech mice for use by the subjects, and two ethernet cards and the connecting cabling. Prerequisite software for the SlavePC are DOS 6.2 and the DOS extensions from the Microsoft Windows (3.11) for workgroups software. No dedicated extra network software is needed, as will be explained later. The software for the SlavePC was completely written in Microsoft Macro Assembler (MASM). The standard software for the MasterPC is Windows 95. Its dedicated software is written in Visual Basic and can run under Excel 97 or as a stand-alone application compiled with Visual Basic 5. Although several specific software packages are available, such as MEL (Micro Experimental Laboratory; Schneider, 1988) or ERTS (Experimental RunTime System; Dutta, 1995), we chose to write our own software, because of the specific needs of the present experiment. As was already mentioned, the present dual-task experiment required that audiovisual material from a videotape be presented simultaneously with CRT task stimuli.

The choice of MASM (instead of C program language) is inspired by the need for direct control of the hardware from the SlavePC and is based on the standard book for system programming on PCs, PC 5 Intern (Tischer \& Jennich, 1996). For reasons of readability and usability, we intend to rewrite the major part of this software in $\mathrm{C}$ in the future. In the present experiment, however, there was no need for a higher programming language for the $486 \mathrm{PC}$, because the data reduction was done on the MasterPC.

\section{SOFTWARE FOR THE SLAVEPC}

The software for the SlavePC consists of timing routines (millisecond accuracy), screen control, mouse data decoding, and synchronization of the computer software with the start of the videotape. The SlavePC is configured as a data logger. Central to the software is the timing routine that is activated every millisecond. Because this PC has no hard disk, the software and the file with the timing data for the visual stimuli are loaded from the hard disk of the MasterPC through the network. The whole file with the timing data is loaded at once in RAM memory. This file contains the exact moments and stimuli types (**, O or $\mathrm{X}$ ) that need to be projected. Every millisecond, the timer routine checks the data and executes the necessary commands. At the same time, there is a check for incoming mouse data. Both the start of the stimuli and the mouse events are logged, and the actual timer count is added. It is essential that the time of the visual stimuli be logged, because of the possible delay of a few milliseconds between the intended and the actual start of the stimulus. This delay is caused by the screen refresh routine that synchronizes the images with the vertical traceback. The exact moment of every mouse click can be calculated in comparison with the start of the stimulus image on the screen. To prevent unnecessary disk access overload, all the data are kept in RAM and put on file 
on the hard disk of the MasterPC at the end of the experiment. This time stamping technique is also used in MEL, as was described by Schneider, Zuccolotto, and Tirone (1993). The method is inspired by the multiparameter method, a technique used in large nuclear experiments that also requires that there be no further data processing during the experiment. As such, different transformations of the data are possible afterwards, and there is no need for a higher processing language in the SlavePC. Although the software for the SlavePC is intended to be controlled by the MasterPC, it can be used stand-alone. A menu option is therefore foreseen that enables the user to start the software with or without waiting for the start tone from the video. The required data file (DATAIN.TXT) with the timing data for the visual stimuli is a standard text file, which can be made with a simple text editor. Each line has the following format: IMG TYPE TICS LENGTH. IMG consists of one letter- $\mathrm{W}$, L, or R-and indicates whether the warning, the left stimulus $(X)$, or the right stimulus $(\mathrm{O})$ has to be displayed. Type (F or T) indicates whether the length of the stimulus (LENGTH) is given in frames or milliseconds. TICS is the start moment of the image in milliseconds. In our case, the warning is the double star $\left({ }^{* *}\right)$. The left stimulus is the $\mathrm{X}$ (for which the subject needs to respond by clicking the left mouse button), and the right stimulus is the $O$. The leftright sequences, the time between the warning signal and the stimulus, and the time between two consecutive warning signals are generated at random in the VBA program on the MasterPC with the use of the Visual Basic "rnd()" function. Although the sequence is fully random for 1 subject, they are the same for all the subjects.

The output data file (DATAOUT.TXT) consists of lines with the following format: EVNTTYPE INFO TIMING. EVENTTYPE is the type of event (MOUSE1, MOUSE2, IMG, BEGIN, or END); INFO indicates the mouse status or stimulus type. For example: "MOUSE1 LRUD 5600" indicates that the right mouse button of mouse 1 was pressed at $5.6 \mathrm{sec}$; "IMG L 5600" indicates that the left stimulus was put on the screen at 5.6 sec.

The problem of millisecond accuracy on a PC has repeatedly been discussed in the literature. As was mentioned by a number of authors (e.g., Graves \& Bradley, 1987), it is not possible to process a fast RT with standard software on a PC. Even the timer control of the latest version of Visual Basic 5 on a Pentium 200 still lacks the necessary accuracy for timing control under $50 \mathrm{msec}$. We used the method described by Brysbaert, Bovens, D'Ydewalle, and Van Calster (1989), Bührer, Sparrer, and Weitkunat (1987), Crosbie (1989), and Heathcote (1988). The approach consists of the modification of the timer chip (8253) that controls the time-of-day clock. We checked to make sure that this did not influence the working of the network software.

The use of a computer screen for projecting visual stimuli in tachistoscope experiments is described by Fin- ley (1989) and Graves and Bradley (1988). In order to obtain a stable screen image, the update needs to be synchronized with the refresh rate of the monitor. Therefore, the method of virtual screens (Finley, 1989) is used. With a normal VGA card, it is possible to select two or more RAM buffers that contain the screen data. In our software, one screenpage (A) is filled with "black" pixels during startup. This page is put permanently on the screen. As such, the computer projector seems to be dimmed and does not "bother" with the projection of the videotape. The moment one image goes off screen, the succeeding image is loaded into the second screen RAM buffer (B). The very moment the image needs to be projected, the status of the screen is checked. When the display is in its next vertical traceback period, buffer B is made the active screen buffer. As such, the visual stimulus (a white image on a black background) is projected, starting from the top of the screen. At the end of the stimulus, both RAM buffers are switched back in the same way, and the screen is "black" again. Because the screen refresh takes place every $14 \mathrm{msec}$, a warning stimulus of roughly $50 \mathrm{msec}$ can be obtained by displaying it for four frames. Since the shortest time gap between two stimuli is $600 \mathrm{msec}$ in the present experiment, there is plenty of time for the software to put the next image into the nonactive screen buffer. Problems concerning screen refreshing on computers have already been described by Dlhopolsky (1989). The method of screen switching is also mentioned by Finley and is used in tachistoscope experiments, as was described by Graves and Bradley (1988). The method is described in detail by Paredes, Miller, and Creeger (1990). Our software is based on procedures mentioned in PC 5 Intern.

As was mentioned by Crosbie (1990) and Segalowitz and Graves (1990), a computer serial mouse is an appropriate instrument for RT measurements on a $\mathrm{PC}$, but the problem is the lack of speed. A standard serial mouse transmits its data to the PC at 1,200 baud. For every mouse click, three or four bytes are transmitted, which results in $30-40 \mathrm{msec}$ for one mouse click. Therefore, the time between two consecutive mouse clicks needs to be a minimum of $30-40 \mathrm{msec}$ in order to be detected. In our case, this problem of minimal RT resolution could affect the measure of time between the moment at which the subject releases the central mouse button and the moment at which the subject presses another one (MT). MT for all test subjects varied between 300 and $600 \mathrm{msec}$. We therefore concluded that the minimal resolution of $40 \mathrm{msec}$ between two mouse clicks was sufficient.

The time accuracy should, however, be much better with our method. Beringer (1992) and Segalowitz and Graves (1990) measured the RT of the Microsoft and the NCE serial mouse with special hardware as approximately $2 \mathrm{msec}$. We assume that their results are also true for the Logitech mouse. Although it is possible to use the game port of the PC to obtain faster RTs, we did not use 
this method. First, the accuracy is sufficient, and second, the use of switches connected with the PC needs special equipment for measuring the bouncing quality of the switches (Crosbie, 1990). As was mentioned by Segalowitz and Graves (1990), it is necessary to suppress unneeded mouse movement activity. We therefore removed the rubber mouse ball.

It is not possible to obtain time information from the tape with a standard video recorder. However, as we mentioned before, in the present dual-task experiment, it was necessary to know the moment of the beginning of the videotape exactly. A small beep was therefore put at the beginning of the tape, a few seconds before the actual start of the tape. The audio output of the video was then connected through an audio amplifier to one of the input datalines of the standard parallel port of the SlavePC. The beep of the video was detected in the computer as a series of 0-1-0 transitions. This signal is analyzed by the software in the computer. It is rejected if it does not have the necessary repetition $(750 \mathrm{kHz})$ or duration $(0.1 \mathrm{sec})$. As such, noise during the startup of the tape is ignored.

\section{SOFTWARE FOR THE MASTERPC}

The software consists of three parts written in Visual Basic. The first part consists of two programs. The first program is for generating the timing data files for the experiment and the training sessions; the second program transforms the stimuli images from .BMP files into a special format that is more easily usable in the assembler program for the SlavePC. The second part is the program for controlling the experiment and the training sessions. The third part is used after the experiment for converting the data for further processing with statistical packages. It consists of two programs. The first program is for converting the data files of the SlavePC into RT and MT values for every response from the subjects; the second program is for merging the data of all the subjects together and calculating mean RT and MT values for the stimuli and responses that were recorded during the sequences of the video display. The program is kept as modular as possible. All the codes for every program block are kept in a single .BAS file. This module can be used as an Excel 97 or Visual Basic 5 module. Every Visual Basic 5 program can be run from the DOS command prompt, with the necessary parameters supplied as command arguments. If they are started directly from Windows 95 or used in Excel 97, the arguments are taken from a text file, EXP98.INF, which must reside in the directory indicated by the MS-DOS environment variable EXP98. In order to be able to control the experiment, an extra form is needed for the visualization of the status of the experiment and for the display of a report about the subjects' percentages of good and bad mouse clicks. Because forms in Excel 97 and Visual Basic 5 are not fully exchangeable, a form is supplied for both environments. Programming in Visual Basic was chosen because it is an easy language and Excel and Visual Basic are widely accepted. It is a very powerful language, fulfilling all the requirements for state-of-the-art programming. Another advantage is the compatibility with Office 98 on Mac. Converting the program for the use on Mac computers should be possible.

\section{SYNCHRONIZATION OF THE DATA TRANSFER BETWEEN BOTH PCS}

We aimed at keeping the software in the SlavePC as simple as possible. All of the software is written for MSDOS. The network software is the one from Windows for workgroups (DOS extension). The startup software is limited to one diskette with MS-DOS and the network software. No hard disk is used in the SlavePC, so there is no need for data transfer between the two PCs. The data are prepared in a subdirectory on the MasterPC that is visible on the SlavePC through the network as a network disk - s:, in our case - from which it can be read directly into the SlavePC. After every experiment, the experimental data gathered by the SlavePC are put on the same disk and can be read by the software in the MasterPC. The locking semaphore for data transfer between the two PCs is kept simple and is implemented by using dummy files for the synchronization of both PCs. When both PCs are operative, all locking files are deleted. The SlavePC must be started first. On this PC, a batch file "G.BAT" is started that waits until the MasterPC creates the file named "PING." This waiting procedure is just a simple DOS batch loop with an "IF EXIST ..." command, as is shown in the Appendix. The loop runs until it detects the existence of the "PING" file. To prevent too much overload in the network, a little program, "COUPLEWT.EXE," is inserted in the loop in order to block the PC every cycle for $500 \mathrm{msec}$. This program runs for about $500 \mathrm{msec}$ before it stops. The program EXP98 is then started on the MasterPC. This program generates a small batch file, "GO.BAT." In this file, the environment variable " $A$ " is assigned a value that corresponds with the command that is to be executed in the program "COUPLE98.EXE" for the SlavePC, which is placed in the second line of "GO.BAT." The file "PING" is then created. As soon as the batch program "G.BAT" in the SlavePC detects the "PING" file, the "GO.BAT" file is started, the software on the SlavePC reads the value of the " $\mathrm{A}$ " variable, and the corresponding action is started. The SlavePC software then creates the "PRESENT" file. As soon as the EXP98 program in the MasterPC detects this file, it signals the operator that all the software is running. In a similar way, the creation of the file "_WAIT" signals that the mice are initialized. The Slave $\overline{P C}$ then waits for the beep from the tape. Once the beep is detected, the SlavePC software creates another file, "BEEPOK," which signals the operator that the experiment has begun. A similar sequence signals the operator that the experi- 
ment is ended. Although this procedure is not very fast, it is sufficient for this kind of experimental setup, and there is no need for writing dedicated network software. From the software on the MasterPC, other tasks can be executed just by changing the value of the DOS variable "A" in the "GO.BAT" file.

\section{DISCUSSION}

Although the system described above was created for a dedicated purpose, it is easily applicable to other environments. The dedicated software for the SlavePC, written in assembly language, can be used for other RT experiments. The program can be used with or without the synchronization beep from the video. It can also be used for any application in which mouse reactions need to be logged. Modifications should only require the creation of other images and the necessary timing text files for the duration and sequence of the images. All the programs can be used freely and downloaded from a Web site (http:// twiprof1 .rug.ac.be/exp 98.html). For every program written in Assembler, Excel 97, and Visual Basic 5, sources and precompiled versions, as well as the necessary data file for demonstration, are available.

Despite its flexibility, the system has some restrictions that should be kept in mind in further developments. First, in every session there can only be one couple. Efficiency should be augmented, if more than one couple is to watch the video at the same time without influencing each other. Because of the modularity of the SlavePC software and the simple network protocol, the system can easily be expanded without much reprogramming of the assembly language software. Second, there is the necessity of having two computer projectors. This may restrict the availability of suitable projection rooms. In our case, only large, uninviting university rooms could be used. If the visual stimuli can be mixed directly on the videotape, there would be no need for a second projector, and any projection room with a video recorder and projector could be used. This is almost impossible with traditional video-editing apparati, because the stimuli have to be put on tape within the precision of a single frame and everything has to be done manually. So if anything changes in the timing sequence of the 150 stimuli, the whole job has to be done over again. This task should, however, be possible, if digital video editing on computer is used. At this moment, we are investigating the possibilities of Adobe Premiere and a MIRO DC30 plus video capture card.

\section{CONCLUSION}

Although this system is designed for a dedicated experiment setup, the software in the SlavePC, acting as an intelligent controller, is kept as general as possible. Any sequence in time of a series of static images that need to be synchronized with another video can be programmed just by creating another data file. Any sequence of mouse clicks from a maximum of two mice connected with one $\mathrm{PC}$ can be logged. The images can be changed just by designing other bitmap (BMP) files. To use it in a different experiment, the software in Visual Basic needs to be adjusted, but we assume that this would require less computer skill than writing assembly language software. In any setting in which multimedia rooms are generally available, the implementation of the present system should require almost no extra hardware costs.

\section{REFERENCES}

BERINGER, J. (1992). Timing accuracy of mouse response registration on the IBM microcomputer family. Behavior Research Methods, Instruments, \& Computers, 24, 486-490.

Brysbaert, M., Bovens, N., D'Ydewalle, G., \& Van Calster, J. (1989). Turbo Pascal timing routines for the IBM microcomputer family. Behavior Research Methods, Instruments, \& Computers, 21, 73-83.

Bührer, M., Sparrer, B., \& WeitKunat, R. (1987). Interval timing routines for the IBM PC/XT/AT microcomputer family. Behavior Research Methods, Instruments, \& Computers, 19, 327-334.

Buysse, A., De ClercQ, A., Roeyers, H., \& Verhofstadt, L. (1999). Decrements in romantic partners' processing of relationshipthreatening information: Impaired processing capacity or motivated distraction? Manuscript submitted for publication.

Crosbie, J. (1989). A simple Turbo Pascal 4.0 program for millisecond timing on the IBM PC/XT/AT. Behavior Research Methods, Instruments, \& Computers, 21, 408-413.

Crosbie, J. (1990). The Microsoft mouse as a multipurpose response device for the IBM PC/XT/AT. Behavior Research Methods, Instruments, \& Computers, 22, 305-316.

DLhopolsKY, J. G. (1989). Synchronizing stimulus displays with millisecond timer software for the IBM PC. Behavior Research Methods, Instruments, \& Computers, 21, 441-446.

DutTA, A. (1995). Experimental RunTime System: Software for developing and running RT experiments on IBM-compatible PCs. Behavior Research Methods, Instruments, \& Computers, 27, 516-519.

FinLEY, G. P. (1989). Tachistoscopic software for the Hercules display controller. Behavior Research Methods, Instruments, \& Computers, 21, 387-390.

Graves, R., \& Bradley, R. (1987). Millisecond interval timer and auditory RT programs for the IBM PC. Behavior Research Methods, Instruments, \& Computers, 19, 30-35.

Graves, R., \& Bradley, R. (1988). More on millisecond timing and tachistoscope applications for the IBM PC. Behavior Research Methods, Instruments, \& Computers, 20, 408-412.

HEATHCOTE, A. (1988). Screen control and timing routines for the IBM microcomputer family using a high-level language. Behavior $R e-$ search Methods, Instruments, \& Computers, 20, 289-297.

JENSEN, A. R., \& MunRo, E. (1979). Reaction time, movement time, and intelligence. Intelligence, 3, 121-126.

Palya, W. L., \& Walter, D. E. (1993). A powerful, inexpensive experiment controller or IBM PC interface and experiment control language. Behavior Research Methods, Instruments, \& Computers, 25 , 127-136.

Palya, W. L., Walter, D. E., \& ChU, J. Y. M. (1995). An inexpensive 1-millisecond experiment control interface for IBM PCs and its userfriendly control language. Behavior Research Methods, Instruments, \& Computers, 27, 129-130.

Paredes, D. R., Miller, K. F., \& Creeger, C. (1990). Graphic precision: Controlling stimulus displays on IBM PC-compatible computers. Behavior Research Methods, Instruments, \& Computers, 22, 319-322.

SCHNEIDER, W. (1988). Micro Experimental Laboratory: An integrated system for IBM PC compatibles. Behavior Research Methods, Instruments, \& Computers, 20, 206-217. 
Schneider, W., Zuccolotto, A., \& Tirone, S. T. (1993). Timestamping computer events to report .1-msec accuracy of events in the Micro Experimental Laboratory. Behavior Research Methods, Instruments, \& Computers, 25, 276-280.

Segalowitz, S. J., \& Graves, R. E. (1990). Suitability of the IBM XT, $\mathrm{AT}$, and PS/2 keyboard, mouse, and game port as response devices in reaction time paradigms. Behavior Research Methods, Instruments, \& Computers, 22, 283-289.
Stelmack, R. M., Houlihan, M., \& McGarry-Roberts, P. A. (1993). Personality, reaction time, and event-related potentials. Journal of Personality \& Social Psychology, 65, 399-409.

TISChER, M., \& JeNNich, B. (1996). PC 5 intern. Brussels: Easy Computing N.V.

Walter, D. E., \& Palya, W. L. (1984). An inexpensive experiment controller for stand-alone applications or distributed processing networks. Behavior Research Methods, Instruments, \& Computers, 16, 125-134.

\section{APPENDIX}

The following is a listing of the G.BAT file that is started on the SlavePC after the network software is loaded and the virtual disk s: linked from the MasterPC.

@echo off

s:

cd $\backslash \exp 98$

del ping

cls

:loopping

couplewt

if not exist ping goto loopping

call go

if not exist stop goto loopping

rem option e exits and puts the screen in text mode

set $\mathrm{A}=\mathrm{e}$

couple98

set $\mathrm{A}=$

Listing of the GO.BAT file created in EXP98 on the MasterPC

SET $A=G$

couple98

All other listings are available from http://twiprofl .rug.ac.be/exp98.html

(Manuscript received October 12, 1998;

revision accepted for publication February 9, 1999.) 